\title{
Molecular physics of a polymer engineering instability: experiments and computation
}

\author{
D. G. Hassell and M. R. Mackley \\ Department of Chemical Engineering, University of Cambridge, CB2 3RA, UK \\ M. Sahin* and H. J. Wilson ${ }^{\dagger}$ \\ Mathematics Department, University College London, Gower Street, London WC1E 6BT, UK
}

\author{
O. G. Harlen ${ }^{1}$ and T. C. B. McLeish ${ }^{2}$ \\ ${ }^{1}$ School of Mathematics; ${ }^{2}$ Polymer IRC, School of Physics \&3 Astronomy, University of Leeds, Leeds LS2 9JT, UK
}

\begin{abstract}
Entangled polymer melts exhibit a variety of flow instabilities that limit production rates in industrial applications. We present both experimental and computational findings, using flow of monodisperse linear polystyrenes in a contraction-expansion geometry, which illustrate the formation and development of one such flow instability. This viscoelastic disturbance is first observed at the slit outlet and subsequently produces large-scale fluid motions upstream. A numerical linear stability study using the molecular structure based Rolie-Poly model confirms the instability and identifies important parameters within the model, which gives physical insight into the underlying mechanism. Chain stretch was found to play a critical role in the instability mechanism, which partially explains the effectiveness of introducing a low-molecular weight tail into a polymer blend to increase its processability.
\end{abstract}

PACS numbers: $47.15 . F e$ 47.20.Gv 47.50.Gj 47.57.Ng 83.50.Uv 83.60.Wc

There are numerous types of experimentally observed instability in polymer melt flow; a recent review [1] highlights three forms observed in extrusion that occur at increasing rates of flow. The first two are "sharkskin" instabilities, which develop due to free surface effects, and "stick-spurt" or "stick-slip" instabilities, which result from material compression and stick-slip at the wall. While the mechanisms underlying the formation of these first two are relatively well understood $[2,3]$, the third class, termed "volume instability", is less so [4]. This instability in converging flows for extrusion and injection moulding has been known for many years, and an empiricism has been developed. But as yet there is no understanding of the underlying physics of the problem, and the inherent connection between the viscoelastic instability and the molecular polymer dynamics. Understanding the underlying physical process would greatly enhance industry's ability to define efficient processing conditions.

This letter outlines recent work in which we created an idealised model flow, related to the engineering flows in that the essential elements are present, but simplified so that the experimental variables are well-controlled and so the whole flow field can be modelled. We have use molecularly well-characterised materials (building on previous work for monodisperse materials under tightly controlled flow conditions $[5,6]$ ) so that the connection between viscoelastic properties and molecular structure can be maintained without empirical fitting. Through these careful

\footnotetext{
* Current address: Department of Aerospace Engineering Sciences, University of Colorado, Boulder, Colorado, 80309, USA

${ }^{\dagger}$ Electronic address: helen.wilson@ucl.ac.uk
}

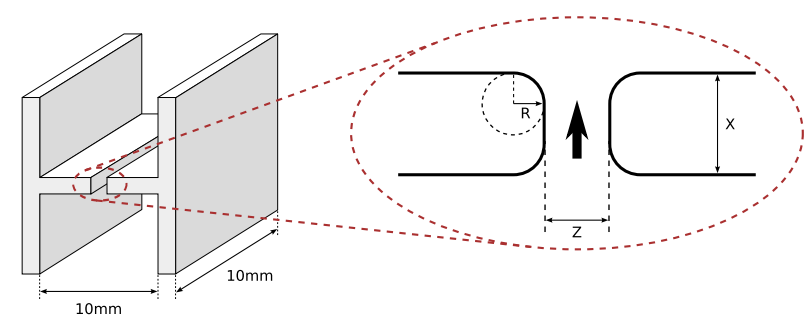

FIG. 1: A schematic illustration of the experimental geometry used in this work.

experiments and multiscale modelling we have elucidated the mechanism of instability.

These experiments were performed in a Multi-Pass Rheometer [7] using the rounded 7:1:7 contractionexpansion slit geometry outlined in Fig. 1. The narrowed region of the slit has length $X=1.5 \mathrm{~mm}$ and width $Z \approx 1.4 \mathrm{~mm}$, with rounded corners of radius $R=0.375 \mathrm{~mm}$. The upstream and downstream regions are $10 \mathrm{~mm}$ square in cross-section.

Flow-induced birefringence (FIB) was used to identify the transient development of stress for five materials with molecular weight $(\mathrm{Mw})$ ranging from $110-523 \mathrm{k}$. The flow instability, shown in Fig. 2, was observed for the three highest molecular weight materials, and was similar to that previously observed in bright field [6]. It is seen to originate at the slit outlet and propagate back upstream over time.

Analysis of the experimental work was performed to investigate the critical parameters for instability using Weissenberg numbers to characterise both the material molecular chain stretch and molecular orientation within the flow. This dimensionless number is defined as the apparent wall shear rate $\dot{\gamma}=6 Q / Z^{2} D$, where $Q$ is the 
(a)
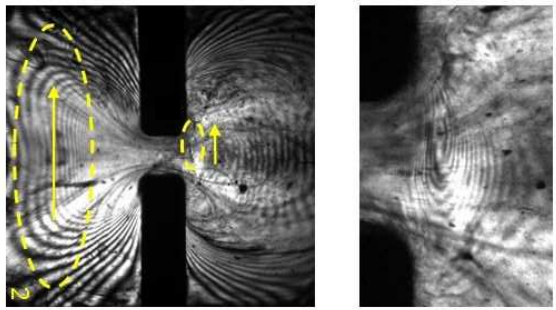

(b)
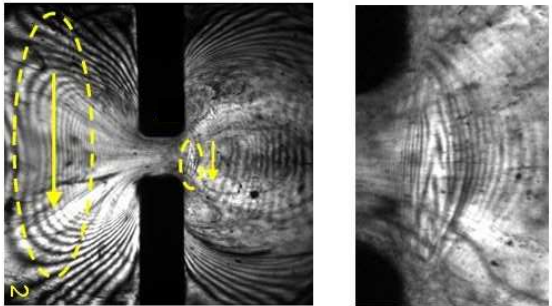

Flow
FIG. 2: FIB images of the $488 \mathrm{k} \mathrm{Mw}$ material at $180^{\circ} \mathrm{C}$, illustrating elements of the flow instability. The images are presented at two sequential times (a) and (b), half an oscillation period apart. At each time a magnified downstream image is shown on the right. Static photographs alone do not capture the form of the instability well; the detailed form is much more evident from observing a video sequence. The disturbance is first observed downstream of the contraction, and manifests as an oscillation of the fringe pattern perpendicular to the bulk flow; this is characterised by lateral movement of the elliptical zero stress eye, seen in the images on the right. It then propagates upstream and influences the upstream stress pattern, producing oscillations similar to those seen downstream. Flow is from left to right. Apparent wall shear rate $\dot{\gamma}=3.6 \mathrm{~s}^{-1} ;$ Weissenberg numbers $W e_{R}=1.6, W e_{d}=73$.

volumetric flow rate, multiplied by either the Rouse time $\left(\tau_{R}\right)$ for molecular chain relaxation, $W e_{R}=\dot{\gamma} \tau_{R}$ or the reptation time $\left(\tau_{d}\right)$ for molecular orientation relaxation, $W e_{d}=\dot{\gamma} \tau_{d}$. The ratio of these numbers, $\tau_{d} / \tau_{R}$, is a function of the polymer molecular weight. A Weissenberg number above one corresponds to chain stretch (for $W e_{R}$ ) or molecular orientation (for $W e_{d}$ ) within the flow. In this study flow instabilities were only observed at flow rates where both Weissenberg numbers were above one. Temperature was used as a variable parameter to process the different molecular weight materials at similar $\tau_{d}$ and $\tau_{R}$ to determine any clear trend for instability onset in relation to these two relaxation times. Example results are shown in Fig. 3, which plots the parameter space of molecular weight, represented by $\tau_{d} / \tau_{R}$, and the material deformation defined by $W e_{R}$. It illustrates the region in which instabilities are observed, and clearly highlights that for low ratios of $\tau_{d} / \tau_{R}$ the instabilities are not observed even at high rates of deformation in this flow geometry. From this and a similar plot of $\tau_{d} / \tau_{R}$ against $W e_{d}$, there is no simple criterion for instability in terms

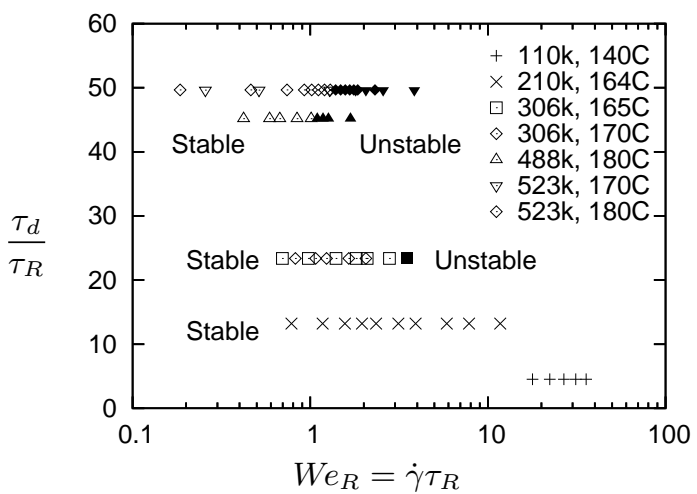

FIG. 3: Processing stability map of the ratio of relaxation times with respect to Rouse time Weissenberg number highlighting the parameter space in which instabilities were observed. Solid symbols represent unstable flows.

of one critical Weissenberg number $W e_{R}$ or $W e_{d}$.

Numerical modelling focussed on the linear stability properties of the flowing system, in which the base flow was assumed to be two-dimensional and stability to three-dimensional perturbations was studied. Although linear stability is not guaranteed to identify all instabilities (some viscoelastic instabilities are inherently nonlinear [8]), experiments suggest that the melt instability we are pursuing does have linear onset. We model the polymer using the Rolie-Poly model [9], currently the most advanced differential formulation of the DoiEdwards tube model for linear polymer melts that is also compact enough to be computable in finite element complex flow calculations. It incorporates at the level of two modes per chain the processes of reptation, convective constraint release (CCR) [10], chain stretch and retraction. We use a multimode version incorporating a solvent viscosity term (without inertia):

$$
\begin{gathered}
\boldsymbol{\nabla} \cdot \boldsymbol{u}=0 ; \quad-\boldsymbol{\nabla} p+\eta \nabla^{2} \boldsymbol{u}+\sum_{i} G_{i} \boldsymbol{\nabla} \cdot \boldsymbol{\sigma}_{i}=0 \\
D \boldsymbol{\sigma}_{i} / D t=\boldsymbol{\kappa} \cdot \boldsymbol{\sigma}_{i}+\boldsymbol{\sigma}_{i} \cdot \boldsymbol{\kappa}^{\top}-\tau_{d, i}^{-1}\left(\boldsymbol{\sigma}_{i}-\boldsymbol{I}\right) \\
\quad-2 \tau_{R, i}^{-1}\left(1-S_{i}^{-1 / 2}\right)\left(\boldsymbol{\sigma}_{i}+\beta^{*} S_{i}^{-1 / 2}\left(\boldsymbol{\sigma}_{i}-\boldsymbol{I}\right)\right)
\end{gathered}
$$

in which $\boldsymbol{u}$ is the fluid velocity, $p$ pressure, $\boldsymbol{\kappa}$ the velocity gradient, and $3 S_{i}$ is the trace of the polymer stress tensor $\boldsymbol{\sigma}_{i}$ for each mode. The physical parameters are $\eta$ (effective solvent viscosity), $G_{i}$ (modulus of each mode), $\tau_{R, i}$ (Rouse time for each mode), $\tau_{d, i}$ (reptation time for each mode) and $\beta^{*}$ (CCR parameter).

We considered three levels of numerical modelling for each material: $[\mathbf{A}]$ the simplest model, a single RoliePoly mode with no solvent viscosity; [B] a single RoliePoly mode with solvent viscosity; and [C] solvent viscosity plus two Rolie-Poly modes. In each case the values 
of the physical parameters were obtained, as in earlier studies [5, 11], from fitting to the full Likhtman-McLeish model [12] of the chosen molecular weight linear polymer.

The steady base flow was found using a semi-staggered finite volume method, similar to earlier work [13] but using Newton's method rather than time-dependent calculation. If the underlying flow is unstable, time-dependent simulations will never find a steady state. Similar timedependent calculations have been carried out by Alves \& coworkers using the PTT model [14]. To calculate the stability properties of this solution, we linearised equations (1-2) for small (lower case) perturbations about the (upper case) base solution $\Phi(x, y, z, t)=\Phi_{0}(x, y)+$ $\phi(x, y) \exp [i k z+\omega t]$. The solution vector $\Phi$ consists of all the flow variables $\left\{\boldsymbol{u}, p, \boldsymbol{\sigma}_{i}\right\}$. The linearisation results in a generalized eigenvalue problem for $\phi(x, y)$ and $\omega$ :

$$
\omega \phi(x, y)=\mathcal{L}\left(\Phi_{0}(x, y) ; \partial_{x}, \partial_{y}, k\right) \phi(x, y)
$$

in which $\mathcal{L}$ is a linear operator acting on the perturbation $\phi$. This is a standard technique; Smith et al. [15] have applied it to viscoelastic systems using a finite-element solver for the base flow. We solve the linear system by the shift-invert Arnoldi method, looking for the eigenvalues with largest real part, and the system is unstable if one or more eigenvalue has a positive real part. The numerical method is described more fully in [16]; the results we present here are robust to mesh refinement. The period of oscillation of an unstable mode is given by $2 \pi / \operatorname{Im}(\omega)$ and the wavelength in the out-of-plane direction by $\lambda=2 \pi / k$.

Both the experimental 7:1:7 and benchmark 8:1:8 contraction-expansion geometries were modelled using a two-dimensional mesh, and their results are essentially equivalent. In each case the inlet and outlet regions have length $17 \mathrm{~mm}$ with periodic boundary conditions connecting the two ends. The highest molecular weight material $\left(523 \mathrm{k} \mathrm{Mw}\right.$, with $[\mathbf{A}]$ relaxation times $\tau_{R} \approx 1 \mathrm{~s}$ and $\tau_{d} \approx 53 \mathrm{~s}$ at $170^{\circ} \mathrm{C}$ ) was used as a case study material for numerical modelling. Its parameters $[\mathbf{C}]$ are:

$$
\begin{aligned}
G_{1} & =1.132 \times 10^{5} \mathrm{~Pa} & G_{2} & =42250 \mathrm{~Pa} \\
\tau_{d, 1} & =27.1 \mathrm{~s} & \tau_{d, 2} & =2.17 \mathrm{~s} \\
\tau_{R, 1} & =0.4907 \mathrm{~s} & \tau_{R, 2} & =0.1356 \mathrm{~s} \\
\eta & =9894 \mathrm{Pas} & \beta^{*} & =0.283
\end{aligned}
$$

With this technique an instability was found at a reptation Weissenberg number $\dot{\gamma} \tau_{d, 1}=120$, corresponding to an apparent wall shear rate of $\dot{\gamma} \approx 4.4 \mathrm{~s}^{-1}$. The same experimental instability was observed at an apparent wall shear rate of $\dot{\gamma} \approx 1.9 \mathrm{~s}^{-1}$. Partial explanations for this discrepancy are that the numerical study does not exactly capture the lowest unstable flow rate $\dot{\gamma}_{c}$ but instead simply demonstrates instability at some flow rate $\dot{\gamma}>\dot{\gamma}_{c}$; or from three-dimensional effects (as the numerical study assumes a channel of infinite depth).

We shifted all timescales and moduli to the experimental temperature of $180^{\circ} \mathrm{C}$ by standard WLF shifting, using WLF parameters for PS taken from the literature $[17,18]$; with this method the instability onset at $180^{\circ} \mathrm{C}$ is predicted to be at $\dot{\gamma} \approx 12 \mathrm{~s}^{-1}$ compared with the experimental observation of $\dot{\gamma} \approx 3.6 \mathrm{~s}^{-1}$; note that time-temperature superposition does not work perfectly on the experimental critical flow rate (the result at $170^{\circ} \mathrm{C}$ would suggest a critical apparent wall shear rate at $180^{\circ} \mathrm{C}$ closer to $\dot{\gamma} \approx 9.8 \mathrm{~s}^{-1}$ ) so we would not expect a perfect match between idealised numerical calculations and the experiments. Nonetheless these results are impressive: no artifical adjustments of parameters have been made here, and these calculations are really $a b$ initio predictions. However, stability results can depend in subtle ways on constitutive details [19] so it is still possible that our computational results are specific to the Rolie-Poly equation. Nonetheless, our prediction of critical flow rate is relatively good, particularly at $170^{\circ} \mathrm{C}$, as is the form of the unstable flow, as we shall see later.

The wavelength of the numerically calculated unstable perturbation in the out-of-plane direction is $\lambda=44 \mathrm{~mm}$, much longer than any of the characteristic lengthscales of the problem, including the experimental slit depth. In essence, we would expect to see exactly the same mode of instability if we were to consider a perturbation entirely in the plane of flow (equivalent to a perturbation having infinite $\lambda$ and hence two-dimensional flow). This makes it unlikely that the mechanism of instability is related to the interaction of normal stresses with curved streamlines [20], as that well-characterised instability is fully three-dimensional. A recent paper by Alves \& Poole [21] uses scaling laws based on the curved streamline mechanism to explain a steady, purely twodimensional "divergent flow" phenomenon in a smooth contraction-expansion flow; we believe that the oscillatory instability we observe is qualitatively different and derives from a different physical mechanism.

Fig. 4(a) shows streamlines for the perturbation flow just above the critical $W e$. The shading represents the flow in the direction of the base flow: this is asymmetric across the slit, indicating a circulation region just downstream of the contraction. It is the effect of this circulation on the zero-birefringence point in the flow that causes the experimentally observed "side-to-side" motion.

The period of oscillations predicted by the numerical study at $170^{\circ} \mathrm{C}$ is around $19 \mathrm{~s}$, which corresponds well with the value of $\approx 13 \mathrm{~s}$ seen just above the critical experimental Weissenberg number. The flow-component of the extra stress from the first Rolie-Poly mode, which is the dominant mode in this flow, is illustrated for the unstable perturbation in Fig. 4(b). This, too, is antisymmetric across the channel, and the largest changes in both stress and vertical velocity due to the instability occur just downstream of the contraction, where the experimental instabilities are first observed. From this we conclude that the instabilities observed by our numerical technique are physical, as they relate directly to the experimental results.

In order to elucidate the mechanism of the instability, the computational code was applied to a simpler [B] 
(a)

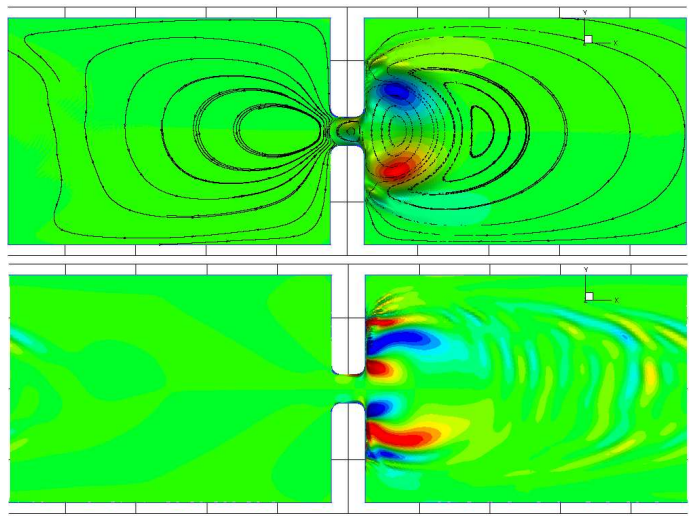

FIG. 4: The unstable perturbation flow for the two-modes plus solvent Rolie-Poly model of the $523 \mathrm{k} \mathrm{Mw}$ fluid at $170^{\circ} \mathrm{C}$, with $\dot{\gamma} \tau_{d, 1}=120$ and $k=0.1$. (a) Streamlines, and shading representing the velocity in the principal flow direction; note the strong circulation region just downstream of the contraction. (b) Plot of the perturbation to the $x x$ component of the first (dominant) Rolie-Poly mode. Note again the dominant region downstream of the contraction.

version of the $523 \mathrm{k}$ Mw fluid:

$$
\begin{aligned}
& G=1.104 \times 10^{5} \mathrm{~Pa}, \quad \eta=3.070 \times 10^{4} \mathrm{Pas}, \\
& \tau_{d}=27.1 \mathrm{~s}, \quad \tau_{R}=0.678 \mathrm{~s}, \quad \beta^{*}=1,
\end{aligned}
$$

for which no instability was found. Probing the numerical parameter space revealed that instability could be provoked either by increasing $\tau_{d} / \tau_{R}$ by a factor of 20 (consistent with the trends of Fig. 3, but which would correspond to a much higher $\mathrm{Mw}$ than the first unstable experimental material) or (much more realistically) by reducing the CCR parameter $\beta^{*}$ to its $[\mathbf{C}]$ value of 0.283 . This observation leads us to believe firstly that, in line with the experiments, this instability is inherent to entangled melts - i.e. to those with stretch and orientation processes on different timescales. Secondly, we believe that the critical physical phenomenon for instability is the amount of chain stretch (whose relaxation is enhanced by decreasing $\tau_{R}$ or by increasing the convective constraint release by increasing $\beta^{*}$ ). This mechanism is supported by the experimental observations that materials with a low ratio $\tau_{d} / \tau_{R}$, in which relatively little chain stretch occurs, are stable even at high flow rates. If the molecular weight is too low, such that the chain stretch relaxes on a timescale similar to orientation; or in the presence of too much convective constraint release, then the observed instability does not manifest itself. These results are a step towards a physical explanation for the effectiveness of introducing a low-molecular weight additive into a polymer to increase its processability [6]. These molecules effectively increase convective constraint release of longer molecular weight chains thereby reducing their chain stretch at a specific flow-rate. The use of molecular constitutive equations of increasing sophistication together with stability analysis of complex flow geometries will enable rational process design to avoid unstable viscoelastic flows.

\section{Acknowledgments}

DGH and MRM thank Simon Butler for technical assistance and Lian Hutchings and Dow for materials. HJW and MS acknowledge the use of UCL Research Computing facilities (Altix). All authors thank John Embery and Richard Graham. This work was funded through the $\mu \mathrm{PP}^{2}$ project, EPSRC GR/T11807/01.
[1] J.-F. Agassant, D. R. Arda, C. Combeaud, A. Merten, H. Munstedt, M. R. Mackley, L. Robert, and B. Vergnes, IPP 21, 239 (2006).

[2] A. Allal, A. Lavernhe, B. Vergnes, and G. Marin, JNNFM 134, 127 (2006).

[3] M. Ranganathan, M. R. Mackley, and P. H. J. Spitteler, J. Rheol. 43, 443 (1999).

[4] C. Combeaud, Y. Demay, and B. Vergnes, JNNFM 121, 175 (2004).

[5] M. W. Collis, A. K. Lele, M. R. Mackley, R. S. Graham, D. J. Groves, A. E. Likhtman, T. M. Nicholson, O. G. Harlen, T. C. B. McLeish, L. R. Hutchings, et al., J. Rheol. 49, 501 (2005).

[6] M. W. Collis and M. R. Mackley, JNNFM 128, 29 (2005).

[7] M. R. Mackley, R. T. J. Marshall, and J. B. A. F. Smeulders, J. Rheol. 39, 1293 (1995).

[8] B. Meulenbroek, C. Storm, V. Bertola, C. Wagner, D. Bonn, and W. van Saarloos, PRL 90, 024502 (2003).

[9] A. E. Likhtman and R. S. Graham, JNNFM 114, 1 (2003).

[10] G. Marrucci, JNNFM 62, 279 (1996).
[11] J. Bent, L. R. Hutchings, R. W. Richards, T. Gough, R. Spares, P. D. Coates, I. Grillo, O. G. Harlen, D. J. Read, R. S. Graham, et al., Science 301, 1691 (2003).

[12] A. E. Likhtman and T. C. B. McLeish, Macromolecules 35, 6332 (2002).

[13] M. Sahin and H. J. Wilson, JNNFM 147, 79 (2007).

[14] M. A. Alves, P. J. Oliveira, and F. T. Pinho, JNNFM 122, 117 (2004).

[15] M. D. Smith, R. C. Armstrong, R. A. Brown, and R. Sureshkumar, JNNFM 93, 203 (2000).

[16] M. Sahin and H. J. Wilson, JNNFM (2008), doi:10.1016/j.jnnfm.2008.01.004.

[17] J. D. Ferry, Viscoelastic Properties of Polymers (Wiley, 1980).

[18] A. E. Likhtman, Macromolecules 38, 6128 (2005).

[19] A. M. Grillet, A. B. Bogaerds, G. W. M. Peters, F. P. T. Baaijens, and M. Bulters, J. Rheol. 46, 651 (2002).

[20] P. Pakdel and G. H. McKinley, PRL 77, 2459 (1996).

[21] M. A. Alves and R. J. Poole, JNNFM 144, 140 (2007). 